\title{
The Ras effectors NORE1A and RASSF1A are frequently inactivated in pheochromocytoma and abdominal paraganglioma
}

\author{
Janos Geli, Nimrod Kiss, Fredrik Lanner ${ }^{1}$, Theodoros Foukakis, \\ Natalia Natalishvili², Olle Larsson ${ }^{2}$, Per Kogner ${ }^{3}$, Anders Höög ${ }^{2}$, \\ Geoffrey J Clark ${ }^{4}$, Tomas J Ekström ${ }^{5}$, Martin Bäckdahl, Filip Farnebo ${ }^{1}$ \\ and Catharina Larsson
}

\footnotetext{
Department of Molecular Medicine and Surgery, CMM L8:01, Karolinska Institutet, Karolinska University Hospital Solna, SE-171 76 Stockholm, Sweden,

${ }^{1}$ Department of Cell and Molecular Biology, Karolinska Institutet, SE-171 77 Stockholm, Sweden,

${ }^{2}$ Department of Oncology-Pathology, Karolinska Institutet, Karolinska University Hospital Solna, SE-171 76 Stockholm, Sweden,

${ }^{3}$ Department of Woman and Child Health, Karolinska Institutet, Karolinska University Hospital Solna, SE-171 76 Stockholm, Sweden,

${ }^{4}$ Department of Cell and Cancer Biology, National Cancer Institute, Rockville, Maryland, USA and

${ }^{5}$ Department of Clinical Neuroscience, Karolinska Institutet, Karolinska University Hospital Solna, SE-171 76 Stockholm, Sweden

(Requests for offprints should be addressed to J Geli; Email: janos.geli@ki.se)
}

\begin{abstract}
NORE1A (RASSF5) and RASSF1A are newly described Ras effectors with tumour suppressor functions. Both molecules are frequently inactivated in various cancers. In this study, we aimed to explore the potential involvement of NORE1A and RASSF1A in pheochromocytoma and abdominal paraganglioma tumorigenesis. A panel of 54 primary tumours was analysed for NORE1A and RASSF1A mRNA expression by TaqMan quantitative RT-PCR. Furthermore, NORE1A and RASSF1A promoter methylation was assessed by combined bisulphite restriction endonuclease assay and methylation-sensitive Pyrosequencing respectively. The anti-tumorigenic role of NORE1A was functionally investigated in Nore1A-transfected PC12 rat pheochromocytoma cells by fluorescent inhibition of caspase activity and soft agar assays. Significantly suppressed NORE1A and RASSF1A mRNA levels were detected in primary tumours compared with normal adrenal medulla $(P<0.001)$. Methylation of the NORE1A promoter was not observed in primary tumours. On the other hand, $9 \%(5 / 54)$ of the primary tumours examined showed RASSF1A promoter methylation greater than $20 \%$ as detected by Pyrosequencing. Methylation of the RASSF1A promoter was significantly associated with malignant behaviour $(P<0.05)$. Transient expression of Nore1a resulted in enhanced apoptosis and impaired colony formation in soft agar. Our study provides evidence that NORE1A and RASSF1A are frequently suppressed in pheochromocytoma and abdominal paraganglioma. Silencing of NORE1A contributes to the transformed phenotype in these tumours.
\end{abstract}

Endocrine-Related Cancer (2007) 14 125-134

\section{Introduction}

The Ras family consists of small GTP-binding proteins with an established role in basic cellular functions related to growth, differentiation and apoptosis. Upon activation, Ras molecules can contribute to tumorigenesis or to cellular tumour defence, apparently diverse effects that are likely related to the downstream interacting Ras effector molecules (Frame \& Balmain
2000, Feig \& Buchsbaum 2002). Several recent reports suggest the involvement of Ras effector molecules in neoplasia by the loss of normal growth inhibitory effects (Feig \& Buchsbaum 2002, Agathanggelou et al. 2005).

NORE1A (RASSF5) is a member of the Ras association domain family (RalGDS/AF-6; Tommasi et al. 2002, Hesson et al. 2003), and the gene locus 
is located in the chromosomal region 1q32.1. Data from experimental model systems suggest that NORE1A can act as a tumour suppressor by inducing apoptosis and delaying cell cycle progression in various cancer cell lines (Khokhlatchev et al. 2002, Vos et al. 2003, Aoyama et al. 2004). NORE1A is often silenced in various human neoplasias, and promoter methylation has been demonstrated as the main underlying mechanism (Tommasi et al. 2002, Chen et al. 2003, Hesson et al. 2003, Vos et al. 2003). So far, NORE1A gene mutations have not been identified in primary tumours or cancer cell lines (Tommasi et al. 2002, Chen et al. 2003, Hesson et al. 2003). Deletions in $1 \mathrm{q}$ are not frequent events in human cancer.

The closely related gene family member RASSF $1 A$ is located in $3 \mathrm{p} 21.3$, a frequently deleted region in human neoplasias (Lerman \& Minna 2000). RASSF1A is a bona fide tumour suppressor (Burbee et al. 2001, Dreijerink et al. 2001, Kuzmin et al. 2002, Chow et al. 2004, Hesson et al. 2004, Tommasi et al. 2005). RASSF1A inactivation has been observed in different tumour types, the most frequent way of inactivation being promoter hypermethylation (Hesson et al. 2004, Agathanggelou et al. 2005, Dammann et al. 2005). RASSF1A has been shown to heterodimerize with NORE1A (Ortiz-Vega et al. 2002) and mediate pro-apoptotic signals via recruitment of the pro-apoptotic mammalian Ste20 related kinase (MST1) (Khokhlatchev et al. 2002, Praskova et al. 2004), suggesting that NORE1A and RASSF1A are involved in the same pro-apoptotic pathway.

Pheochromocytomas and abdominal paragangliomas (the latter are here referred to as paraganglioma and in the literature often as extra-adrenal pheochromocytoma) are neuroendocrine tumours arising from neural crest-derived chromaffin cells. Pheochromocytomas are present in the andrenal medulla, whereas paragangliomas occur along the paravertebral and para-aortic sympathetic paraganglia. Pheochromocytomas frequently exhibit loss of RASSF1A expression by promoter methylation or deletion (Astuti et al. 2001, Dammann et al. 2005). To our knowledge, no study has investigated the involvement of NORE1A gene in these tumour entities. The current study was undertaken to examine the role of NORE1A and RASSF1A in pheochromocytoma and paraganglioma. Gene expression and promoter methylation status were assessed and evaluated in relation to various clinicopathological characteristics. Furthermore, we have enquired whether re-expression of Norela in PC12 pheochromocytoma cells can reverse the tumorigenic phenotype.

\section{Materials and methods}

\section{Tumours and reference samples}

Informed consent was obtained from all patients and the local ethics committee at the Karolinska University Hospital approved the study of the tissue material. Patients were operated at the Endocrine Surgical Unit at the Karolinska University Hospital Solna (Edström Elder et al. 2003). The study includes 56 tumours: 46 primary pheochromocytomas, 8 primary paragangliomas and 2 paraganglioma distant metastases. The clinical data are detailed for each case in Supplementary Table A which can be viewed online at http://erc.endocrinologyjournals.org/supplemental/. Cases 2 and 19 as well as cases 14 and 27 are paired primary tumours and metastases. Seven tumours are from patients with a known familial form of the disease including multiple endocrine neoplasia type 2 (cases 11, 17 and 40), neurofibromatosis type 1 (cases 12, 23 and 41) and von Hippel Lindau disease (case 29). Before the analyses, representative sections were cut from all frozen tumour specimens and histopathologically evaluated to confirm the high proportion of tumour cells $(>70 \%)$. Tumours were classified according to the criteria of the US Armed Forces Institute of Pathology, i.e. the diagnosis of malignancy required an extensive local invasion and/or distant metastasis (Lack 1997).

The following samples were used as normal controls for NORE1A and RASSF1A mRNA expression measurements: pooled human RNA from healthy adrenal glands (NA pool $_{\text {p }}$ of 61 Caucasian subjects deceased of sudden death, purchased from Clontech Laboratories, Inc., Mountain View, CA, USA (reference number: 636528, ages 15-61), and adrenal medullary RNA (Norm 1-8) derived from eight Caucasian individuals (ages 50-76), who had no medical history of neoplastic disease and died due to cancer-unrelated causes (Clinomics Biosciences, Inc., Watervliet, NY, USA). For the methylation analyses, DNA derived from three histopathologically verified normal adrenal tissue specimens were used (Norm 9-11). Two of these patients were subsequently diagnosed with benign cystic lesions without hormonal abnormalities, and one was diagnosed with a small adrenocortical adenoma with surrounding normal adrenal tissue.

\section{Real-time quantitative PCR (qRT-PCR)}

Total RNA was extracted using RNeasy RNA extraction kit (Qiagen) and quantified by spectrophotometry. RNA integrity was confirmed by electrophoresis in $1 \%$ denaturing agarose gels, whereby the $18 S$ and $28 S$ bands were clearly demonstrated. Total 
RNA $(1.5 \mu \mathrm{g})$ was reverse transcribed in $100 \mu \mathrm{l}$ reactions using High-Capacity cDNA Archive Kit (Applied Biosystems, Foster City, CA, USA), following the manufacturer's instructions. qRT-PCR was performed using ABI PRISM 7700 Sequence Detection System (Applied Biosystems). Gene-specific primers and probes available as TaqMan Gene Expression Assays (Applied Biosystems) were used, including assays Hs00417514_m1 for NORE1A and Hs00945257_m1 for RASSF1A. Sixty-five nanograms of each sample were amplified in a TaqMan $2 X$ Universal Master Mix (final concentration $1 \times$ ) under the following conditions: $50{ }^{\circ} \mathrm{C}$ for $2 \mathrm{~min}$ and $95^{\circ} \mathrm{C}$ for $10 \mathrm{~min}$, followed by 40 cycles of $95^{\circ} \mathrm{C}$ for $15 \mathrm{~s}$ and $60{ }^{\circ} \mathrm{C}$ for $1 \mathrm{~min}$. To select the most appropriate endogenous controls, we tested four different housekeeping genes across the samples: glyceraldehyde-3phosphate dehydrogenase (GAPDH), $\beta$-actin $(A C T B)$, $\beta$-2-microglobulin $(B 2 M)$ and hypoxanthine guanine phosphoribosyltransferase 1 (HPRTl). Three of these showed relatively stable expression across the tumour samples and were selected for further analysis: HPRTI (Hs99999909_m1), ACTB (Hs99999903_m1) and B2M (Hs00187842_m1). All qRT-PCR experiments were performed in duplicate on two different occasions and included no-template controls. Serial dilutions of $\mathrm{NA}_{\text {pool }}$ cDNA were amplified in parallel to establish a standard curve for relative quantification.

The raw data were analysed using Sequence Detection System (SDS 1.9.1, Applied Biosystems) and quantified in relation to the generated standard curves. The expression values of each target gene were then normalized to the geometrical average of the three housekeeping genes (HPRT1, ACTB and B2M) and subsequently to the reference $\mathrm{NA}_{\text {pool }}$, which was assigned a nominal value of 1 .

\section{DNA extraction and bisulphite treatment}

High molecular weight DNA was extracted using standard procedures with proteinase $\mathrm{K}$ digestion, phenol-chloroform extraction and ethanol precipitation. All bisulphite treatment was done using EZ DNA Methylation Kit (Zymo Research Corporation, Orange, CA, USA), following the manufacturer's instructions.

\section{Combined bisulphite restriction endonuclease assay (COBRA)}

The NORE1A promoter was amplified from bisulphitetreated DNA of tumours and normal controls (Norm 910) by nested PCR as described (Chen et al. 2003). PCR products were purified using Microcon YM-100 (Millipore Corporation, Billerica, MA, USA) and cleaved by TaqI (Invitrogen Life Technologies, Inc.). Genomic DNA from MCF-7 cells was used as a positive control for the methylated state (Chen et al. 2003), while human leukocyte DNA from healthy individuals served as an unmethylated assay control. DNA from Norm 9-10 was used as a healthy reference. Results were confirmed by repeated analyses on two different occasions.

\section{Quantification of RASSF1A methylation by Pyrosequencing}

Bisulphite-treated DNA from tumours and normal controls (norm 11) were used for PCR amplifications. Pyrosequencing was performed using the PSQHS96 system (Biotage AB, Uppsala, Sweden) including PyroGold reagents. Primers were designed by Biotage $\mathrm{AB}$ and are available at the PyroMark Assay Database (Biotage $\mathrm{AB}$ ). The primers amplify a stretch of the RASSF1A exon 1 (Ensemble ID: ENST00000266020). The outer primers target CpG-free regions within this stretch. PCR was carried out using $1.5 \mu \mathrm{l}$ bisulphitetreated DNA under the following conditions: $95^{\circ} \mathrm{C}$ for $15 \mathrm{~min}, 45 \times\left(95^{\circ} \mathrm{C}\right.$ for $20 \mathrm{~s}, 55^{\circ} \mathrm{C}$ for $20 \mathrm{~s}, 72^{\circ} \mathrm{C}$ for $20 \mathrm{~s}), 72^{\circ} \mathrm{C}$ for $10 \mathrm{~min}, 4^{\circ} \mathrm{C}$. Intactness of the PCR product was assessed by electrophoresis on $3 \%$ agarose gel and subsequent ethidium bromide staining. Pyrosequencing was carried out at Biotage $\mathrm{AB}$. The Pyrosequencing primers target a 24-nt segment, which lies within the previously amplified stretch of RASSF1A exon1 and contains five CpGs (CCGCCCGGCCCGCGCTTGCTAGCG). This segment was chosen to cover the sites analysed for RASSF 1A promoter methylation in previous reports (Honorio et al. 2003).

\section{Transfection of PC12 cells with Nore1a constructs}

Generation, sequencing and characterization of rat NORE1A (Norela) cDNA constructs have previously been published (Vos et al. 2003). Briefly, Nore la cDNA was cloned into pcDNAF, a modified version of pcDNA3 (Invitrogen Life Technologies, Inc.), which attaches a FLAG tag to the insert (pcDNAF Nore1a). To generate the green fluorescent protein $(\mathrm{GFP}) \sim$ Norela construct rat, Nore la cDNA was inserted into a Clontech's GFP vector (Clontech Laboratories, Inc.). The correct sizes of the inserts were confirmed by the restriction endonuclease cleavage followed by agarose gel electrophoresis. PC12 rat pheochromocytoma cells were grown using standard reagents and procedures. Transient transfections were carried out with GFP Norela and pcDNAF $\sim$ Nore1a using LipofectAmine 2000 
(Invitrogen Life Technologies, Inc.), according to the manufacturer's instructions. PC12 cells were transiently transfected with pcDNAF Nore $1 \mathrm{a}$ and GFP at a molar ratio of 10:1 or GFP Nore1a. Medium was changed after $12 \mathrm{~h}$. Successful transfection was confirmed after $24 \mathrm{~h}$ by fluorescent microscopy and western blot analyses (data not shown).

\section{Apoptosis assay}

To determine the fraction of apoptotic cells after transfection, Sulphorhodamine Apoptosis Detection Kit Caspase Assay was used (Immunochemistry Technologies LLC, Bloomington, MN, USA). Briefly, cells were harvested $24 \mathrm{~h}$ after transfection, resuspended in the culture medium and analysed by fluorescent inhibition of caspase activity (FLICA), according to the manufacturer's instructions. Cells were analysed with a FACScalibur (BD Biosciences, San Jose, CA, USA) flowcytometer. The green fluorescent cell population, i.e. transfected cells, was assessed for red fluorescence (FLICA), indicating binding to active caspases.

\section{Soft agar assay}

Twenty-four hours after transfection, cells were harvested, fluorescence activated cell sorting (FACS) sorted for green fluorescence and replated for an additional $36 \mathrm{~h}$ to allow recovery from cellular shock caused by FACS. Cells were subsequently harvested, counted and seeded at a density of 1250 cells per well in $0.3 \%$ soft agar, which was overlaid onto $0.6 \%$ bottom agar (Agarose type VII, Sigma-Aldrich). Cells were fed every 4-5 days. After 11 days, cells were stained with MTT (Roche AB) overnight and colonies were counted.

\section{Statistical analyses}

All analyses were performed in STATISTICA data analysis software system version 7 (Statsoft, Inc., Tulsa, OK, USA), and for all tests, $P$-values $\leq 0.05$ were considered significant. Statistical analyses comprised data only from the 54 primary tumour samples, while the two metastases were excluded. Expression values for NORE1A and RASSF1A were compared between groups of samples with different clinical and histopathological characteristics, as well as between tumour samples and normal controls. Mann-Whitney test was used to assess differences in target gene expression between tumours versus normal controls, females versus males, paragangliomas versus pheochromocytomas and benign versus malignant disease. Correlation between tumour size and target gene expression, as well as between age at presentation and target gene expression, was examined using Spearman rank-order correlations. To assess correlations between the degrees of methylation at each of the five $\mathrm{CpG}$ sites within the RASSF 1A promoter tested by Pyrosequencing, and the different clinical and histopathological tumour features, we used MannWhitney test and Spearman rank-order correlations. Furthermore, Spearman rank-order correlations and Kruskal-Wallis analysis were utilized to assess the relationship between $R A S S F 1 A$ promoter methylation and RASSF1A mRNA expression.

\section{Results}

\section{Reduced NORE1A and RASSF1A mRNA expression in pheochromocytoma and paraganglioma}

Significantly reduced NORE1A mRNA levels were detected in primary tumours when compared with normal adreno-medullary control samples $(P=0.00001$; MannWhitney test; Fig. 1A). No significant correlations were detected between NORE1A mRNA levels and the various clinicopathological characteristics.

RASSF1A was substantially under-expressed in primary tumours when compared with normal adrenomedullary control samples $(P=0.000018$; MannWhitney test; Fig. 1B). There was no significant correlation between RASSF1A mRNA levels and benign versus malignant characteristics, tumour type, sex or tumour size (Mann-Whitney test and Spearman

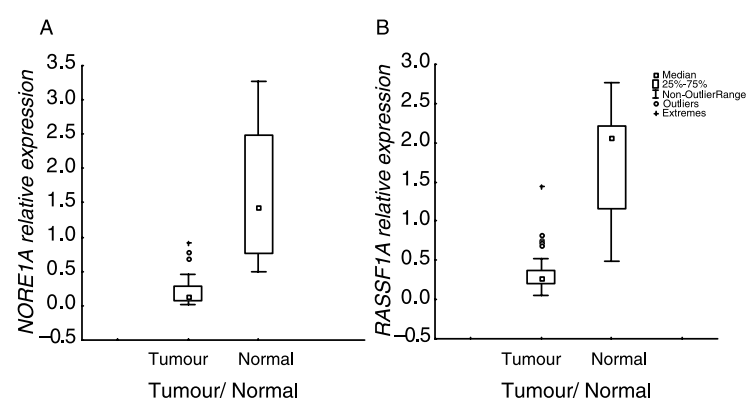

Figure 1 (A) Detection of reduced NORE1A mRNA expression in primary pheochromocytomas and paragangliomas. Nominal value of 1 corresponds to the expression level measured in a pool of total adrenal gland extracts from 61 individuals. The box plot demonstrates that the overall NORE1A mRNA expression in tumours is significantly lower than that in the eight normal adreno-medullary reference samples. (B) Detection of reduced RASSF1A mRNA expression in primary pheochromocytomas and paragangliomas. Values are normalized to the expression level measured in a pool of total adrenal gland extracts from 61 individuals. The box plot demonstrates that the overall RASSF1A mRNA expression in tumours is significantly lower than that in the eight normal adreno-medullary reference samples. 


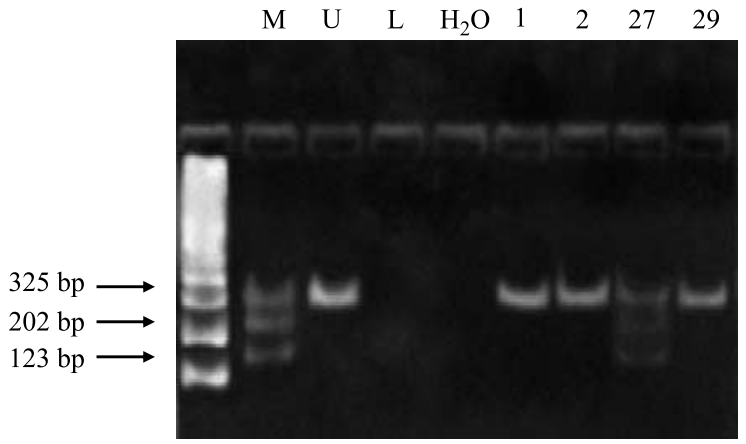

Figure 2 COBRA analysis of the NORE1A promoter. PCR product from bisulphite-treated DNA was cleaved with Taql. The analysed $325 \mathrm{bp}$ of the NORE1A promoter contains 35 $\mathrm{CpG}$ sequences. If this fragment is methylated, the two Taql sites will be cleaved, resulting in the bands of 202, 123 and $10 \mathrm{bp}$. Top panel: MCF-7 (M) cell line was used as a positive control for the methylated status, which clearly demonstrates partial restriction cleavage indicating partial methylation. $U$, human lymphocyte DNA was used as a negative control for the unmethylated status; L, lymphocyte DNA without bisulphite treatment; $\mathrm{H}_{2} \mathrm{O}$, water control. Four representative tumours are shown to the right. Only tumour 27, a distant metastasis, showed modest partial methylation as demonstrated by weak partial cleavage. Tumours 1,2 and 29 are without methylation in the NORE1A promoter. A $123 \mathrm{bp}$ ladder is used as a size marker.

rank-order correlations). However, a tendency towards lower RASSF1A expression was observed in tumours from patients with a younger age at diagnosis ( $P<0.05 ; R=0.29$; Spearman rank-order correlations).

\section{NORE1A promoter methylation is a rare event in pheochromocytoma and paraganglioma}

Methylation of the NORE1A promoter was not detected in the normal adrenal reference samples or in the primary tumours. Weak partial methylation of the NORE1A promoter was observed in one tumour (sample no. 27), which was a metastasis obtained from a patient with malignant paraganglioma (Fig. 2).

\section{Quantification of RASSF1A methylation by pyrosequencing}

The five CpG sites were analysed quantitatively by methylation-sensitive Pyrosequencing (Fig. 3; Table 1). Normal reference adrenal DNA exhibited a low level of methylation (below 10\%) at the $\mathrm{CpG}$ sites assessed. Significantly higher degree of methylation was observed in primary malignant tumours compared with benign ones $(P<0.05$; Mann-Whitney test $)$. For all five $\mathrm{CpG}$ sites, tumours were categorized into groups according to the methylation value, i.e. low methylation, intermediate and high methylation. Groups were then compared with regard to RASSF 1A mRNA expression. No difference was seen between the groups with regard to RASSF1A expression (Kruskal-Wallis analysis). No significant correlation was seen between the methylation degree at the $\mathrm{CpG}$ sites tested and the RASSF1A expression (Spearman rank-order correlation). Both metastases as well as their original primary tumours showed high levels of RASSF1A promoter CpG methylation.

\section{Reduced colony formation after Nore1A trans- fection in PC12 cells}

More than two- and ninefold reduction in colony formation was observed by GFP Nore1a- and pcDNAF $\sim$ Nore1a-transfected PC12 cells respectively when compared with negative controls (GFP-transfected cells; $P<0.05$; Fig. 4).

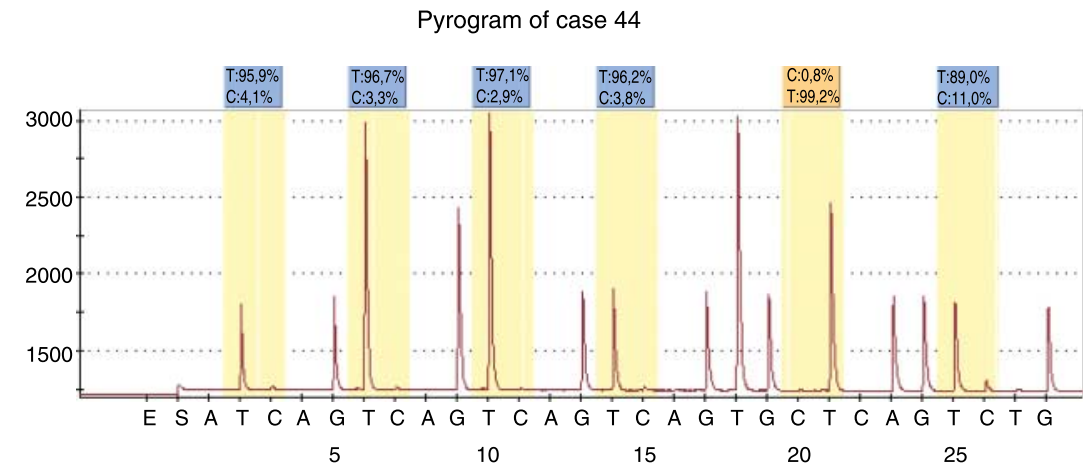

Figure 3 Pyrogram from quantification of methylation by Pyrosequencing of the RASSF1A promoter. During the Pyrosequencing reaction, a ' $C$ ' is incorporated if the template $C p G$ is methylated, while a ' $T$ ' is incorporated if the template $C p G$ is unmethylated. In the resulting pyrogram, the proportion of C:T reflects the degree of methylation at the particular CpG sites assessed. 
Table 1 Results from the studies by qRT-PCR and promoter methylation anlayses

\begin{tabular}{|c|c|c|c|c|c|c|c|c|c|}
\hline \multirow[b]{2}{*}{ Case no. } & \multirow{2}{*}{$\begin{array}{c}\text { RASSF1A } \\
\text { mRNA }\end{array}$} & \multirow{2}{*}{$\begin{array}{c}\text { NORE1A } \\
\text { mRNA }\end{array}$} & \multicolumn{6}{|c|}{ RASSF1A methylation by pyrosequencing } & \multirow[b]{2}{*}{ NORE1A COBRA } \\
\hline & & & Pos 1 & Pos 2 & Pos 3 & Pos 4 & Pos 5 & Average & \\
\hline 1 & 0.205 & 0.305 & 1.3 & 1.7 & 1.5 & 1.1 & 1.4 & 1.4 & Unmeth \\
\hline 2 & 0.21 & 0.32 & 57.9 & 60.6 & 62.2 & 53.5 & 52.6 & 57.36 & Unmeth \\
\hline 3 & 1.44 & 0.14 & 2.6 & 2.7 & 12 & 10.6 & 16.7 & 8.92 & Unmeth \\
\hline 4 & 0.245 & 0.01 & 0 & 2.4 & 0 & 1.1 & 0 & 0.7 & Unmeth \\
\hline 5 & 0.755 & 0.08 & 0 & 1 & 1.2 & 0 & 0 & 0.44 & Unmeth \\
\hline 6 & 0.815 & 0.15 & 10.5 & 7.1 & 5.4 & 7.3 & 12.6 & 8.58 & Unmeth \\
\hline 7 & 0.225 & 0.14 & 3 & 2.1 & 2.1 & 2.8 & 3.3 & 2.66 & Unmeth \\
\hline 8 & 0.22 & 0.455 & 3.1 & 6.6 & 4.3 & 11.6 & 5.4 & 6.2 & Unmeth \\
\hline 9 & 0.155 & 0.03 & 24.5 & 5 & 4.1 & 5.3 & 6.7 & 9.12 & Unmeth \\
\hline 10 & 0.74 & 0.695 & n.d. & n.d. & n.d. & n.d. & n.d. & n.d. & Unmeth \\
\hline 11 & 0.4 & 0.4 & 19.2 & 3.9 & 2.8 & 3.2 & 24.6 & 10.74 & Unmeth \\
\hline 12 & 0.26 & 0.15 & 1.7 & 1.7 & 1.6 & 1.4 & 2.3 & 1.74 & Unmeth \\
\hline 13 & 0.49 & 0.13 & 5.3 & 3.5 & 3.4 & 5 & 6.4 & 4.72 & Unmeth \\
\hline 14 & 0.265 & 0.14 & 66.5 & 68.4 & 40.5 & 57.6 & 58.8 & 58.36 & Unmeth \\
\hline 15 & 0.16 & 0.79 & 2.3 & 3.9 & 12.6 & 11.5 & 3.5 & 6.76 & Unmeth \\
\hline 16 & 0.72 & 0.095 & 1 & 1.2 & 1.2 & 0.9 & 1 & 1.06 & Unmeth \\
\hline 17 & 0.115 & 0.01 & 1.9 & 2 & 1.6 & 1.9 & 2.1 & 1.9 & Unmeth \\
\hline 18 & 0.33 & 0.45 & 5.2 & 2.6 & 2.6 & 3.4 & 29.1 & 8.58 & Unmeth \\
\hline 19 & 0.165 & 1.77 & 50 & 54 & 53.2 & 47.2 & 45.3 & 49.94 & Unmeth \\
\hline 20 & 0.32 & 0.02 & 6.1 & 29.4 & 2.7 & 2 & 2.3 & 8.5 & Unmeth \\
\hline 21 & 0.225 & 0.105 & 1 & 1.3 & 1.5 & 1.1 & 1.3 & 1.24 & Unmeth \\
\hline 22 & 0.28 & 0.185 & 19.2 & 3.8 & 3.1 & 3.2 & 19.7 & 9.8 & Unmeth \\
\hline 23 & 0.275 & 0.36 & n.d. & n.d. & n.d. & n.d. & n.d. & n.d. & Unmeth \\
\hline 24 & 0.215 & 0.125 & n.d. & n.d. & n.d. & n.d. & n.d. & n.d. & Unmeth \\
\hline 25 & 0.27 & 0.11 & 1 & 1.2 & 1.4 & 0.9 & 1.1 & 1.12 & Unmeth \\
\hline 26 & 0.355 & 0.155 & 70.2 & 71.1 & 72.2 & 63 & 61.1 & 67.52 & Unmeth \\
\hline 27 & 0.255 & 0.045 & 69.5 & 72.5 & 72.2 & 64 & 62 & 68.04 & Part meth \\
\hline 28 & 0.375 & 0.265 & 1.7 & 2.6 & 5 & 2.4 & 2.8 & 2.9 & Unmeth \\
\hline 29 & 0.495 & 0.345 & 0 & 1.2 & 1.3 & 0.8 & 0.8 & 0.82 & Unmeth \\
\hline 30 & 0.695 & 0.155 & 24.6 & 2.9 & 2.2 & 2.2 & 20.5 & 10.48 & Unmeth \\
\hline 31 & 0.23 & 0.075 & 3.4 & 3 & 3.9 & 25.7 & 9.8 & 9.16 & Unmeth \\
\hline 32 & 0.22 & 0.25 & 1.2 & 1.4 & 2 & 2.7 & 1.9 & 1.84 & Unmeth \\
\hline 33 & 0.285 & 0.195 & 36.1 & 7.7 & 23.4 & 10.5 & 16.6 & 18.86 & Unmeth \\
\hline 34 & 0.37 & 0.025 & 28 & 3.7 & 3.2 & 1.9 & 2.7 & 7.9 & Unmeth \\
\hline 35 & 0.25 & 0.125 & 0.8 & 1.3 & 1.4 & 0.8 & 0.8 & 1.02 & Unmeth \\
\hline 36 & 0.265 & 0.065 & 1.5 & 1.7 & 1.6 & 1.3 & 1.3 & 1.48 & Unmeth \\
\hline 37 & 0.13 & 0.925 & 0 & 0 & 10.2 & 0 & 17.4 & 5.52 & Unmeth \\
\hline 38 & 0.1 & 0.025 & 5.7 & 5.7 & 10.9 & 6.9 & 10.7 & 7.98 & Unmeth \\
\hline 39 & 0.16 & 0.08 & 7.8 & 5 & 5.5 & 4.4 & 10.8 & 6.7 & Unmeth \\
\hline 40 & 0.15 & 0.23 & 1 & 1.5 & 1.6 & 1 & 1 & 1.22 & Unmeth \\
\hline 41 & 0.21 & 0.285 & 0 & 0 & 0 & 0 & 0 & 0 & Unmeth \\
\hline 42 & 0.045 & 0.015 & 23.4 & 17.4 & 12.2 & 23.3 & 28 & 20.86 & Unmeth \\
\hline 43 & 0.3 & 0.4 & 0 & 1.2 & 1.2 & 0.6 & 0.7 & 0.74 & Unmeth \\
\hline 44 & 0.135 & 0.015 & 4.1 & 3.3 & 2.9 & 3.8 & 11 & 5.02 & Unmeth \\
\hline 45 & 0.4 & 0.125 & 1.5 & 1.6 & 1.3 & 1.1 & 0.8 & 1.26 & Unmeth \\
\hline 46 & 0.21 & 0.14 & 7.4 & 31.1 & 6.9 & 5.3 & 10.3 & 12.2 & Unmeth \\
\hline 47 & 0.5 & 0.08 & 6.7 & 23.3 & 29.4 & 9.1 & 28 & 19.3 & Unmeth \\
\hline 48 & 0.135 & 0.065 & 7.2 & 7.8 & 5.2 & 10 & 29.7 & 11.98 & Unmeth \\
\hline 49 & 0.31 & 0.285 & 0.9 & 1.7 & 1.3 & 0.9 & 1.1 & 1.18 & Unmeth \\
\hline 50 & 0.275 & 0.155 & 5.3 & 2 & 1.9 & 1.8 & 4.1 & 3.02 & Unmeth \\
\hline 51 & 0.515 & 0.455 & 45.7 & 41.4 & 9.9 & 21.3 & 24.4 & 28.54 & Unmeth \\
\hline 52 & 0.325 & 0.18 & 1.7 & 1.9 & 1.8 & 1.7 & 2 & 1.82 & Unmeth \\
\hline 53 & 0.295 & 0.025 & 0 & 0 & 1.9 & 0 & 0 & 0.38 & Unmeth \\
\hline 54 & 0.48 & 0.105 & 20.8 & 5.2 & 3.8 & 3.7 & 2.9 & 7.28 & Unmeth \\
\hline 55 & 0.34 & 0.13 & 9.7 & 28.3 & 7.8 & 18.1 & 25.3 & 17.84 & Unmeth \\
\hline 56 & 0.225 & 0.035 & n.d. & n.d. & n.d. & n.d. & n.d. & n.d. & Unmeth \\
\hline
\end{tabular}

n.d., not determined; Part meth, partially methylated; Unmeth, unmethylated; Pos 1-5, CpG positions. 


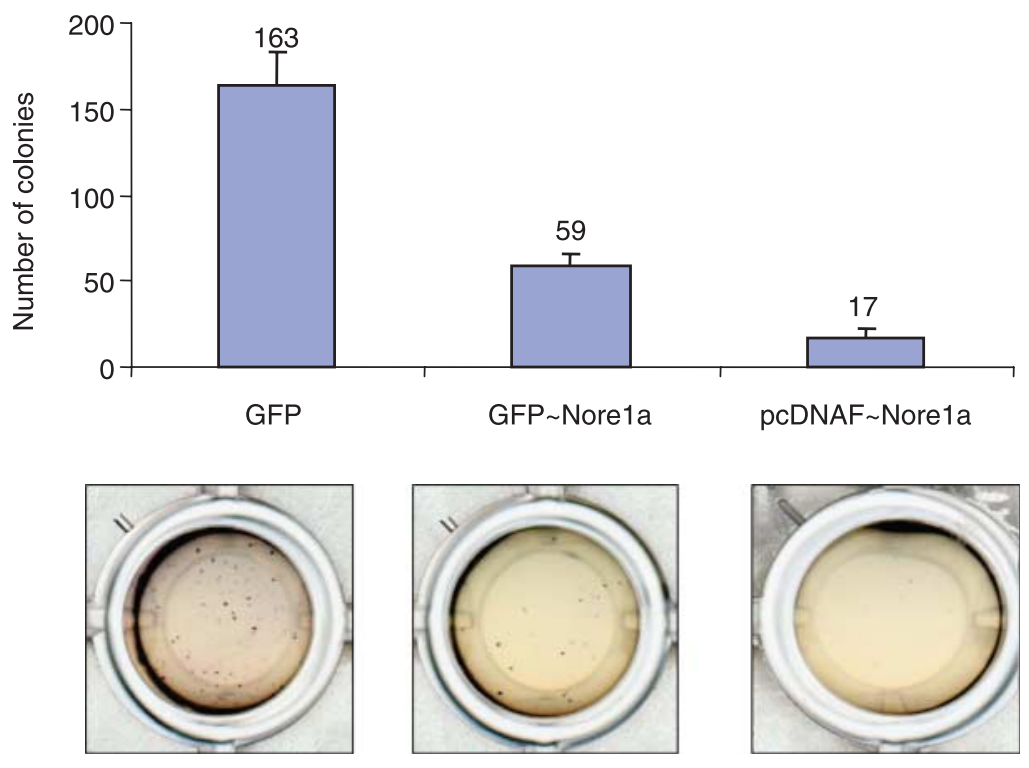

Figure 4 Top: reduced anchorage-independent growth quantified by the number of colonies of PC12 cells after transfection with GFP-Nore1 or pcDNAF Nore1a when compared with only GFP-transfected cells. The results show a composite value from three measurements. Bottom: representative images of soft agar gel assay with corresponding constructs (GFP, GFP Nore1a and pcDNAF Nore1a).

\section{Increased apoptotic rate in PC12 cells transfected with Nore1a}

Transfection of PC12 cells with pcDNAF Nore1a or GFP Nore1a was associated with a 1.5- and 2-fold increase in apoptotic rate respectively when compared with GFP-transfected cells used as negative controls (Fig. 5).

\section{Discussion}

NORE1A is a downstream Ras effector that mediates inhibitory effects with regard to cellular growth and survival. NORE1A can induce apoptosis and cell cycle progression delay in cancer cells (Vos et al. 2003, Aoyama et al. 2004). NORE1A expression is frequently down-regulated or lost in various human cancer cell lines and tumours. Our present observation of markedly decreased NORE1A mRNA expression in pheochromocytoma and paraganglioma is in agreement with previous reports demonstrating suppressed NORE1A expression in other types of neoplasias (Tommasi et al. 2002, Chen et al. 2003, Hesson et al. 2003, Vos et al. 2003, Aoyama et al. 2004).

Several different mechanisms could potentially lead to gene silencing, including structural mutations, deletions, epigenetic modifications and other regulatory mechanisms at the transcriptional or post-transcriptional level. Epigenetic inactivation by promoter methylation has been implicated as a common mechanism underlying NORE1A silencing (Tommasi et al. 2002, Chen et al. 2003, Vos et al. 2003, Hesson et al. 2004). In view of these observations, and our present finding of frequent suppression of NORE1A mRNA expression, we have assessed NORE1A promoter methylation using COBRA assay (Chen et al. 2003). In contrast to results from other tumour types, we have found that NORE1A promoter methylation is a rare event in pheochromocytomas and paragangliomas. Only one tumour, a distant metastasis from a malignant pheochromocytoma, showed slight

FLICA apoptosis assay on Nore1a transfected PC12cells

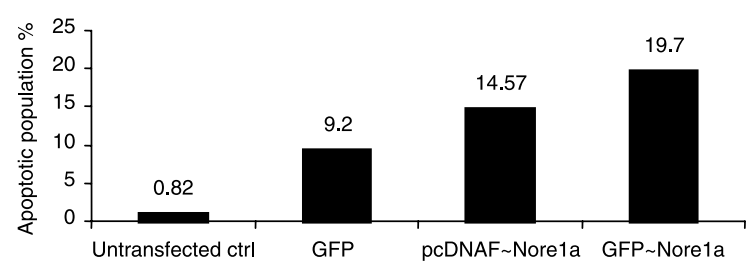

Figure 5 FLICA apoptosis assay of pcDNAF Nore1a- and GFP Nore1a-transfected PC12 cells. Green fluorescent (transfected) cells were gated and further analysed for red fluorescent (FLICA) signal by FACS. Results show that transfection by pcDNAF $\sim$ Nore1a and GFP $\sim$ Nore1a resulted in a 1.5- and 2-fold increase in apoptotic rate respectively when compared with GFP-transfected cells used as negative controls. 
partial methylation. So far, no inactivating mutations in the NOREl gene have been reported in tumours or cancer cell lines (Tommasi et al. 2002, Chen et al. 2003, Hesson et al. 2003, Vos et al. 2003, Hesson et al. 2004). The NORE1 gene is mapped to $1 \mathrm{q} 32.1-2$, a region that is only rarely affected by somatic deletions in human cancer. Our previous comparative genomic hybridisation (CGH) results on pheochromocytomas and paragangliomas showed no indication of major DNA loss on 1q (Edström et al. 2000). Therefore, it does not appear likely that the observed NORE1A transcriptional silencing is due to allelic loss or mutation. This however does not exclude the possibility of smaller allelic losses in the region beyond the resolution of CGH.

Restoration of NORE1A expression in cell lines lacking endogenous expression is associated with reduced cell growth, delayed cell cycle progression, enhanced apoptosis and reduced anchorage-independent growth (Tommasi et al. 2002, Chen et al. 2003, Hesson et al. 2003, Vos et al. 2003, Aoyama et al. 2004). To enquire whether impaired NORE1A expression affects the propensity of pheochromocytoma cells to undergo apoptosis, we have assessed apoptotic rate in Norelatransfected PC12 cells using a fluorescent pan caspase detection system. Increased apoptotic rate was detected amongst transfected cells, indicating that loss of NORE1A expression in pheochromocytomas may impair the cells' ability to perish via programmed cell death, an observation that was also suggested by Vos et al. (2003).

The capacity of tumour cells to grow in an anchorageindependent fashion is a good indicator of their in vivo tumorigenic potential (Cifone 1982). We have investigated the impact of Nore1a on the ability of PC12 cells to proliferate in soft agar. In agreement with other reports, we have observed substantially decreased colony numbers after Nore1a transfection, indicating that impairment of Norela expression may be an important contributing factor to the neoplastic growth of pheochromocytoma cells (Fig. 4; Vos et al. 2003).

The RASSF1A promoter is one of the most commonly hypermethylated tumour suppressor gene promoters in human cancer and its methylation is associated with the loss of RASSF1A gene expression (Dammann et al. 2000, Astuti et al. 2001, Dammann et al. 2001, Murray et al. 2004). Astuti et al. observed 22\% (5/23) of RASSF 1A promoter methylation in sporadic pheochromocytomas (Astuti et al. 2001). In a recent report, Damman et al. detected $48 \%$ methylation $(12 / 25)$ in a series of familial and sporadic pheochromocytomas (Dammann et al. 2005). However, both reports utilized non-quantitative methods, COBRA and methylation specific polymerase chain reaction (MSP) respectively, which could not quantitatively assess the proportion of methylated to unmethylated $\mathrm{CpGs}$ at each of the $\mathrm{CpG}$ sites analysed. This may be particularly important considering that MSP can be sensitive to as little as $0.1 \%$ of methylated alleles (Herman et al. 1996), thus classifying a sample as methylated on the basis of a minor proportion of methylated target, which may not reflect the overall scenario within the tumour sample studied. In the study by Dammann et al. (2005) the cases positive for RASSFIA methylation mainly exhibited partial methylation, i.e. PCR product was detected with both methylated and unmethylated allele-specific primers. Similarly, Astuti et al. (2001) reported only partial digestion in their RASSFIA COBRA analyses in pheochromocytomas, and when DNA from RASSF1A methylated tumours was bisulphite treated and then sequenced, both thoroughly methylated and unmethylated clones were detected. These observations suggest that partial methylation may be attributed to the presence of non-neoplastic stromal cells or heterogeneity within the tumour cell population with regard to RASSF 1A promoter methylation.

In view of these previous findings, we have proceeded to assess the methylation status of the RASSF 1A promoter in a quantitative fashion using methylation-sensitive Pyrosequencing. This technique allows for the relative quantification of methylated $\mathrm{CpGs}$ in relation to unmethylated $\mathrm{CpGs}$ for each of the CpG sites tested. We have found that 9\% (5/54) of the primary tumours examined showed RASSF1A methylation greater than $20 \%$ (as an average of methylation values of the five examined $\mathrm{CpG}$ sites). Three of these five tumours were primary malignant paragangliomas, one was a primary malignant pheochromocytoma and one was a benign pheochromocytoma (Table 1 and Supplementary Table A, which can be viewed online at http://erc.endocrinology-journals.org/supplemental/). Overall, malignant tumours showed significantly higher levels of methylation compared with benign ones $(P<0.05)$. This finding is consistent with other reports showing correlation between the prevalence of RASSF1A promoter hypermethylation and adverse tumour characteristics (Byun et al. 2001, Kuroki et al. 2003, Hesson et al. 2004, Kim et al. 2004). In the two cases of paired primary tumour and metastasis, both the primary lesions and the corresponding metastases showed high levels of methylation. This might suggest that methylation of the RASSF1A promoter is a relatively early event; however, one would need to study an extended number of paired primary tumours and metastases to ascertain this concept.

Pheochromocytomas and paragangliomas in this study displayed significantly suppressed RASSF $1 A$ gene expression levels compared with that measured 
in non-neoplastic adrenal medulla. All samples that showed at least $20 \%$ of methylated alleles for RASSF $1 A$ promoter had greatly reduced expression. Although promoter hypermethylation is the predominant way of RASSF 1A expressional suppression, one may consider other contributing mechanisms of tumour suppressor gene inactivation. Mutation of RASSF1A is rare, although not unprecedented in human cancer (Burbee et al. 2001, Dreijerink et al. 2001, Lo et al. 2001). However, Astuti et al. (2001) did not detect RASSF1A mutation in pheochromocytomas. On the other hand, loss of heterozygosity $(\mathrm{LOH})$ at the $3 \mathrm{p} 21.3$ resident site where RASSF IA is located occurs frequently (38.5\%) in pheochromocytomas (Astuti et al. 2001). Our findings suggest that methylation is not likely to fully account for the thoroughly suppressed RASSF $1 A$ mRNA levels in these tumours. It is thus highly possible that other mechanisms such as $\mathrm{LOH}$ or perturbed transcriptional regulation are significant contributing factors to RASSF1A inactivation in these tumours.

In conclusion, the present study demonstrates that pheochromocytomas and abdominal paragangliomas display markedly suppressed gene expression of two prominent members of the Ras association domain family, NORE1A and RASSF1A, both of which have been widely implicated in the defence against tumorigenic growth. The lack of correlation between the NORE1A/RASSF1A mRNA expression levels and the various clinicopathological features indicates a more general role for these molecules in pheochromocytoma/ paraganglioma tumorigenesis that is not confined to a particular subgroup of patients. RASSF1A but not NORE1A promoter methylation is a frequent event in these tumours, the previous also showing a good correlation with malignant behaviour. Furthermore, we provide functional evidence that reconstitution of Nore 1a expression in $\mathrm{PC} 12$ rat pheochromocytoma cells reduces the tumorigenic phenotype. This study is the first report investigating NORE1A in pheochromocytomas and paragangliomas, as well as revealing an association between the degree of RASSF1A promoter methylation and malignant behaviour in these tumours.

\section{Acknowledgements}

The authors would like to thank Lisa Åhnfalk for expertise in tumour sample handling. The study was supported by the grants from the Swedish Cancer Society, the Göran Gustafsson Foundation for Research in Natural Sciences and Medicine, the Gustav V Jubilee Foundation and the Stockholm County Council. Pyrosequencing was carried out in collaboration with Biotage $\mathrm{AB}$ as detailed in Materials and Methods. No financial or other kind of support was received from Biotage that would compromise this impartiality of this study. The authors declare that there is no conflict of interest that would prejudice the impartiality of this scientific work.

\section{References}

Agathanggelou A, Cooper WN \& Latif F 2005 Role of the Ras-association domain family 1 tumor suppressor gene in human cancers. Cancer Research 65 3497-3508.

Aoyama Y, Avruch J \& Zhang XF 2004 Nore1 inhibits tumor cell growth independent of Ras or the MST1/2 kinases. Oncogene 23 3426-3433.

Astuti D, Agathanggelou A, Honorio S, Dallol A, Martinsson T, Kogner P, Cummins C, Neumann HP, Voutilainen R, Dahia P et al. 2001 RASSF1A promoter region CpG island hypermethylation in phaeochromocytomas and neuroblastoma tumours. Oncogene 20 7573-7577.

Burbee DG, Forgacs E, Zochbauer-Muller S, Shivakumar L, Fong K, Gao B, Randle D, Kondo M, Virmani A, Bader S et al. 2001 Epigenetic inactivation of RASSF1A in lung and breast cancers and malignant phenotype suppression. Journal of the National Cancer Institute 93 691-699.

Byun DS, Lee MG, Chae KS, Ryu BG \& Chi SG 2001 Frequent epigenetic inactivation of RASSF1A by aberrant promoter hypermethylation in human gastric adenocarcinoma. Cancer Research 61 7034-7038.

Chen J, Lui WO, Vos MD, Clark GJ, Takahashi M, Schoumans J, Khoo SK, Petillo D, Lavery T, Sugimura J et al. 2003 The $\mathrm{t}(1 ; 3)$ breakpoint-spanning genes LSAMP and NORE1 are involved in clear cell renal cell carcinomas. Cancer Cell 4 405-413.

Chow LS, Lo KW, Kwong J, To KF, Tsang KS, Lam CW, Dammann R, Huang DPRASSF1A \& Huang DP 2004 RASSF1A is a target tumor suppressor from $3 \mathrm{p} 21.3$ in nasopharyngeal carcinoma. International Journal of Cancer 109 839-847.

Cifone MA 1982 In vitro growth characteristics associated with benign and metastatic variants of tumor cells. Cancer Metastasis Reviews 1 335-347.

Dammann R, Li C, Yoon JH, Chin PL, Bates S \& Pfeifer GP 2000 Epigenetic inactivation of a RAS association domain family protein from the lung tumour suppressor locus 3p21.3. Nature Genetics 25 315-319.

Dammann R, Schagdarsurengin U, Seidel C, Trumpler C, Hoang-Vu C, Gimm O, Dralle H, Pfeifer GP \& Brauckhoff M 2005 Frequent promoter methylation of tumor-related genes in sporadic and men2-associated pheochromocytomas. Experimental and Clinical Endocrinology \& Diabetes 113 1-7.

Dammann R, Yang G \& Pfeifer GP 2001 Hypermethylation of the cpG island of Ras association domain family $1 \mathrm{~A}$ (RASSF1A), a putative tumor suppressor gene from the 3p21.3 locus, occurs in a large percentage of human breast cancers. Cancer Research 61 3105-3109.

Dreijerink K, Braga E, Kuzmin I, Geil L, Duh FM, Angeloni D, Zbar B, Lerman MI, Stanbridge EJ, Minna JD et al. 2001 
The candidate tumor suppressor gene, RASSF1A, from human chromosome $3 \mathrm{p} 21.3$ is involved in kidney tumorigenesis. PNAS 98 7504-7509.

Edström E, Mahlamaki E, Nord B, Kjellman M, Karhu R, Höög A, Goncharov N, Teh BT, Bäckdahl M \& Larsson C 2000 Comparative genomic hybridization reveals frequent losses of chromosomes $1 \mathrm{p}$ and $3 \mathrm{q}$ in pheochromocytomas and abdominal paragangliomas, suggesting a common genetic etiology. American Journal of Pathology 156 651-659.

Edström Elder E, Hjelm Skog AL, Höög A \& Hamberger B 2003 The management of benign and malignant pheochromocytoma and abdominal paraganglioma. European Journal of Surgical Oncology 29 278-283.

Feig LA \& Buchsbaum RJ 2002 Cell signaling: life or death decisions of ras proteins. Current Biology 12 R259-R261.

Frame S \& Balmain A 2000 Integration of positive and negative growth signals during ras pathway activation in vivo. Current Opinion in Genetics \& Development 10 106-113.

Herman JG, Graff JR, Myohanen S, Nelkin BD \& Baylin SB 1996 Methylation-specific PCR: a novel PCR assay for methylation status of CpG islands. PNAS 93 9821-9826.

Hesson L, Dallol A, Minna JD, Maher ER \& Latif F 2003 NORE1A, a homologue of RASSF1A tumour suppressor gene is inactivated in human cancers. Oncogene 22 947-954.

Hesson L, Bieche I, Krex D, Criniere E, Hoang-Xuan K, Maher ER \& Latif F 2004 Frequent epigenetic inactivation of RASSF1A and BLU genes located within the critical 3p21.3 region in gliomas. Oncogene $\mathbf{2 3}$ 2408-2419.

Honorio S, Agathanggelou A, Schuermann M, Pankow W, Viacava P, Maher ER \& Latif F 2003 Detection of RASSF1A aberrant promoter hypermethylation in sputum from chronic smokers and ductal carcinoma in situ from breast cancer patients. Oncogene 22 147-150.

Kim TY, Jong HS, Jung Y, Kim TY, Kang GH \& Bang YJ 2004 DNA hypermethylation in gastric cancer. Alimentary Pharmacology \& Therapeutics 20 (Suppl 1) 131-142.

Khokhlatchev A, Rabizadeh S, Xavier R, Nedwidek M, Chen T, Zhang XF, Seed B \& Avruch J 2002 Identification of a novel Ras-regulated proapoptotic pathway. Current Biology 12 253-265.

Kuroki T, Trapasso F, Yendamuri S, Matsuyama A, Alder H, Mori M \& Croce CM 2003 Promoter hypermethylation of
RASSF1A in esophageal squamous cell carcinoma. Clinical Cancer Research 9 1441-1445.

Kuzmin I, Gillespie JW, Protopopov A, Geil L, Dreijerink K, Yang Y, Vocke CD, Duh FM, Zabarovsky E, Minna JD et al. 2002 The RASSF1A tumor suppressor gene is inactivated in prostate tumors and suppresses growth of prostate carcinoma cells. Cancer Research 62 3498-3502.

Lack EE 1997 Tumours of the Adrenal Gland and ExtraAdrenal Paraganglia. Washington, DC: Armed Forces Institute of Pathology.

Lerman MI \& Minna JD 2000 The 630-kb lung cancer homozygous deletion region on human chromosome 3p21.3: identification and evaluation of the resident candidate tumor suppressor genes. The International Lung Cancer Chromosome 3p21.3 Tumor Suppressor Gene Consortium. Cancer Research 60 6116-6133.

Lo KW, Kwong J, Hui AB, Chan SY, To KF, Chan AS, Chow LS, Teo PM, Johnson PJ \& Huang DP 2001 High frequency of promoter hypermethylation of RASSF1A in nasopharyngeal carcinoma. Cancer Research 61 3877-3881.

Murray PG, Qiu GH, Fu L, Waites ER, Srivastava G, Heys D, Agathanggelou A, Latif F, Grundy RG, Mann JR et al. 2004 Frequent epigenetic inactivation of the RASSF1A tumor suppressor gene in Hodgkin's lymphoma. Oncogene 23 1326-1331.

Ortiz-Vega S, Khokhlatchev A, Nedwidek M, Zhang XF, Dammann R, Pfeifer GP \& Avruch J 2002 The putative tumor suppressor RASSF1A homodimerizes and heterodimerizes with the Ras-GTP binding protein Nore1. Oncogene 21 1381-1390.

Praskova M, Khoklatchev A, Ortiz-Vega S \& Avruch J 2004 Regulation of the MST1 kinase by autophosphorylation, by the growth inhibitory proteins, RASSF1 and NORE1, and by Ras. Biochemical Journal 381 453-462.

Tommasi S, Dammann R, Jin SG, Zhang XF, Avruch J \& Pfeifer GP 2002 RASSF3 and NORE1: identification and cloning of two human homologues of the putative tumor suppressor gene RASSF1. Oncogene 21 2713-2720.

Tommasi S, Dammann R, Zhang Z, Wang Y, Liu L, Tsark WM, Wilczynski SP, Li J, You M \& Pfeifer GP 2005 Tumor susceptibility of Rassf1a knockout mice. Cancer Research 65 92-98.

Vos MD, Martinez A, Ellis CA, Vallecorsa T \& Clark GJ 2003 The pro-apoptotic Ras effector Nore1 may serve as a Ras-regulated tumor suppressor in the lung. Journal of Biological Chemistry 278 21938-21943. 